\title{
Conceptual-Network-Based Philosophy of Science
}

\author{
Bernard Korzeniewski \\ BioSimulation Center, Kraków, Poland \\ Email: bernard.korzeniewski@gmail.com
}

How to cite this paper: Korzeniewski, B. (2019). Conceptual-Network-Based Philosophy of Science. Open Journal of Philosophy, 9, 104-139.

https://doi.org/10.4236/ojpp.2019.92009

Received: March 15, 2019

Accepted: May 5, 2019

Published: May 8, 2019

Copyright $\odot 2019$ by author(s) and Scientific Research Publishing Inc. This work is licensed under the Creative Commons Attribution International License (CC BY 4.0).

http://creativecommons.org/licenses/by/4.0/

\section{c) (i) Open Access}

\begin{abstract}
Conceptual-Network-Based Theory of Science (CNBTS) is presented. It is confronted with the existing philosophies of science rather than with particular scientific theories. The conceptual network, constituting the "substance" of the human psyche, is composed of continuous concepts meaning by connotation. Concepts representing certain aspects of the reality are of completely different nature, than these aspects themselves. Nevertheless, the structure of the conceptual network of science is more or less isomorphic with the structure of the external world (semi-representative realism). The collective conceptual network of science is in a sense a less determined (less precise, more vague) sum, mean or resultant of the individual conceptual networks of particular scientists. During the science development, its conceptual network entwines the physical reality, as a spider web entwines a stony sculpture. The appearance of new theories, paradigms and research programmes consists in a development of new, differently structured fragments (conceptual maps) of the conceptual network of science. Scientific theories and hypotheses cannot be entirely true or false. They can only adhere better or worse to (various aspects of) the external reality, and the progress in science consists in an increase of the degree of this adherence. Hitherto well-working theories cannot be falsified, but only replaced with theories that are more isomorphic with, adhere better to vaster areas of reality.
\end{abstract}

\section{Keywords}

Philosophy of Science, Conceptual Network, Falsification, Representative Realism

\section{Introduction}

None of the existing philosophies of science has been commonly accepted as fully adequate and correctly explaining all important aspects of science develoliu 
ment. Inductionism (Salmon, 1975; Russell, 1912) is not satisfactory as a method (and philosophy) of science for many reasons, but first of all because observations (and all the more experiments) depend on theory as well as on the knowledge and experience of the observer, and as such can be abolished (Chalmers, 1999). In fact, the legitimization of inductionism was already negated by Hume (1975). Logical empiricism (Ayer, ed., 1959), related to inductionism, is so restrictive and stiff by its demand to limit every knowledge to directly observable aspects (facts) of the world by a "typical observer", that it in fact eliminates most scientific theories and thus most of science as we know it. Analytical philosophy (Preston), kin to logical empiricism, shares many of its sins. Early Wittgenstein (1922) identified the structure of language with the structure of our knowledge and cognition of the world, even more, with the structure of (the facts of) the world itself. Falsificationism (Popper, 1968) overlooks the fact that observations and experiments (their interpretations) that are claimed to falsify a given theory can themselves be false, as they depend upon the current knowledge and accepted theories (Chalmers, 1999). In other words, the act of falsification is also prone to falsification. Additionally, all these philosophies do not take into account satisfactorily the complexity of science as a process and relational nature of scientific (and any other) meanings. More realistic descriptions of the process of the development of science were proposed by Kuhn (1962) and Lakatos (1970), who characterize scientific theories as structured entities that guarantee relatively precise meaning of concepts appearing within them. Their ideas are rather similar, although Kuhn emphasizes much more sociological factors affecting science development (therefore, Kuhn represents a more relativistic, while Lakatos-more rationalistic point of view). Research programmes, with their hard core, protective belt and auxiliary hypotheses (Lakatos, 1970) resemble to a significant extent, although are probably more elaborated, than scientific paradigms (Kuhn, 1962). Similarly, the replacement of a degenerative research programme by a progressive research programme (Lakatos, 1970) is analogous to a change of a paradigm during scientific revolution, caused by a crisis, separating two periods of "normal science" (Kuhn, 1962). Nevertheless, also these ideas of scientific paradigms and research programmes fail to display satisfactorily the relational character of science, the interplay between its various elements and the connotative character of the system of meanings formed within it. They also do not deal with the fundamental problem: how scientific theories are created within the minds of scientists and accepted or rejected by scientific societies. Chalmers (1999) postulated unrepresentative realism, according to which different scientific theories apply to different extents to the real world. However, they do not approach the truth in any understanding. Overall, this conception seems at least partly instrumentalist. Additionally, it does not take into account the relational character of the meanings in science, relation between science as a whole, as an intersubjective social phenomenon, and its representations in individual minds of scientists as well as the way in which scientific theories are created by particular scientists and accepted or rejected by scientific society. 
This work is intended to propose a philosophy of science based on the philosophy of conceptual network formulated previously (Korzeniewski, 2010, 2013b, 2015, 2017). Its aim is not to replace the existing philosophies, but rather to supplement and reformulate the most general, best known and most influential of them in order to create a consistent picture of science development, both as a social intersubjective whole and in the brains and subjective minds of particular scientists.

\section{Conceptual Network}

\subsection{General Idea of Conceptual Network}

According to the philosophy of conceptual network formulated before (Korzeniewski, 2010, 2014, 2017), a concept is a certain unit of meaning or sense. Everything that can reach our consciousness (psyche) and constitute its content is either a concept or a complex of concepts. Therefore, a concept can be characterized as a unit of consciousness and thought. For this reason, the idea of "conceptual thinking" has been proposed (Korzeniewski, 2010, 2013b, 2014, 2017), in opposition to Wittgenstein's "linguistic thinking" (Wittgenstein, 1922). A concept comprises all kinds of beings that can be perceived, thought, imagined, or grasped mentally in reality, dream, or in mystical, religious, or narcotic trance by a conscious brain. A flower, Julius Cesar, a category, a centaur, pain, justice, a quantifier - as well as many phenomena so misty and undetermined that they do not have linguistic names attributed to them - all of them are concepts. Concepts correspond not only to individual beings, as for instance linguistic names in a sentence, but also to more complex objects, corresponding to entire sentences, conceptions, and ideas.

The conceptual network has been defined as a complex of concepts that are mutually interconnected by certain determined relations and exhibit some specific properties that fills our consciousness and constitutes the substance of our psyche (Korzeniewski, 2010, 2014, 2017). Concepts in the conceptual network pass smoothly one into another and are not separated by any sharp borderlines. To some extent they resemble hills in a landscape - although we can separate (distinguish) particular hills, there are no sharp limits between them. The conceptual network is a continuous entity, contrary to language, which is composed of discrete names. Two properties decide about the identity of a given concept. First, this is the degree of separation of the sense of a concept, its determination and specification, "intensity" of its meaning in the "semantic field". It results from the fact how many concepts "define" a given concept and decides how clearly and univocally a given concept appears to our consciousness. Second, concepts are defined, characterized and differentiated by the complex of connections between a given concept and other concepts in the conceptual network. This property determines to which concepts and how a given concept it related semantically, which other concepts fix the sense of this concept. In this way the meaning of a given concept is specified. The meaning of a concept is determined 
only and exclusively by its semantic context and its reference to other concepts, and therefore concepts mean by connotation, contrary to the meaning by denotation, where concepts would directly correspond to external (physical) and internal (mental) objects designated by them. The concepts that "define" a given concept are in turn themselves defined by other concepts. Ultimately, all concepts are defined by all other concepts. The entire conceptual network constitutes the proper semantic context for a given concept. Therefore, the philosophy of conceptual network represents a relationistic theory of meaning.

One can define the semantic space with its "dimensions" represented by "significative axes", forming a sort of the Cartesian system of coordinates. In such a space concepts are formed by polarization along significative axes in relation to other concepts. Examples of simple significative axes are: long - short, fast slow, near - distant, red - not red, intelligent - not intelligent. Like concepts, significative axes are continuous objects. Continuous are both the differences "along" axes between their "ends", and the differences "between axes", separating one axis from another. The type of axes polarization (opposed ends of axes) is also determined for a given axis by appropriate concepts. Thus, concepts are determined by significative axes, while significative axes-by concepts. This property constitutes another manifestation of the connotative character of the conceptual network. Senses appear in this network by "layering" or polarization of a "semantic vacuum" along significative axes.

Sensations are equivalent to a direct "activation" by stimuli from the external world (processed by integrative neural structures in the sensory brain cortex, see below) of some already formed concepts in the conceptual network (Korzeniewski, 2010, 2017). This activation can be enhanced by the phenomenon of attention. The existing conceptual network enables interpretation and understanding of incoming signals, constitutes a reference frame for their "mental grasping". On the other hand, stimuli from the external world participate in the formation of new concepts, further determination of the existing concepts and development of the entire conceptual network and its sub-networks (e.g., conceptual maps of scientific theories). Repeating combinations of sensations arriving at the same time can be associated together and form new concepts. These are primary concepts, directly related to particular objects and events in the external (as well as internal) world (Korzeniewski, 2010, 2017). Already formed concepts can also be activated "indirectly", by the autonomous processes of thinking, remembering, dreaming, that is generally by endogenous autonomous activity of the neural network in the brain, underlying the conceptual network (see below). This process can also lead to formation of concepts on a higher level of the hierarchy of generality, the so-called secondary concepts (Korzeniewski, 2010, 2017). They are created by time coincidence of activation of primary concepts as well as by extraction of repeating patterns and structures from them. Secondary concepts represent abstract and general ideas, laws, conceptions and universal beings. Scientific theories are composed of such (complexes of) con- 
cepts. It should be stressed that there is no sharp borderline between secondary and primary concepts, and that several levels of generality and abstractness appear within both these concept categories. The current content of human (self-)consciousness is constituted by the concepts that are just "activated" and from which recurrent signals are received by the cognitive centre in the brain (see below). Incoming sensations are related to the existing concepts, and thus interpreted and understood, adequately processed and possibly incorporated into the conceptual network as new concepts. Particular concepts, especially primary concepts, are activated much stronger by sensory stimuli from the external world than by autonomous processes of thinking, recalling and remembering in the brain. For this reason, we perceive sensations as much clearer and "real" than thoughts, dreams, and recollections. Nothing like discrete, autonomous "pure sensations" or "qualia" (Chalmers, 1997) exists or even has any sense (Korzeniewski, 2010, 2017). The signals coming from the external world (incoming "sensory pictures") mean anything only because they are referred to and interpreted by the already existing conceptual network that entirely determines the "mental content" and "quality" of perceived sensations.

Only a very small fragment of the conceptual network is activated at a given moment and thus can constitute the substance (content) of one's (self-)consciousness (the term "(self-)consciousness" is sometimes used in this work in order to emphasize the fact that the "true", psychical consciousness, as opposed to instrumental consciousness, cannot exist, or have any sense, without self-consciousness). This is related to the phenomenon of attention. This does not change the fact, that there are also unconscious processes of thinking that are not at a given moment "noticed" (grasped) by (self-)consciousness. However, the meaning and understanding of any concept activated at a moment is ultimately based on the entire network. The conceptual network contains better or worse differentiated and internally coherent areas. It is hierarchically organised and contains conceptual sub-networks, maps and sub-maps. There belong to them hierarchically-organized scientific disciplines, religions, worldview, fine arts and literature (their psychical reception), ethics, and the sphere of common (ordinary) concepts (Korzeniewski, 2010, 2014, 2017). In particular, scientific theories constitute conceptual maps and sub-maps within the conceptual sub-network of science.

At the moment of birth humans possess essentially no conceptual network. Nevertheless, a newborn child have at disposal inborn, purely biological significative axes that constitute the reference frame for formation of first concepts from incoming sensations (stimuli received from physical reality and processed by, also inborn, integrative structures, see below) (Korzeniewski, 2010, 2015, 2017). The knowledge about the world of a given person grows during his/her life, the view of the world develops, and the understanding of different aspects of reality, including products of culture, science, and art increases. The meaning of "the same" linguistic names, representing the same aspects of the (external and 
internal) world changes and develops (becomes more complex). Overall, human conceptual network has to evolve during individual (ontogenetic) development. The species Homo sapiens originated in the course of biological evolution from animal ancestors that had much less complex neural systems and brains, and were devoid of (self-)consciousness. Therefore, (self-)consciousness, together with the underlying neural/conceptual network (see below), developed during biological evolution (phylogenesis). Finally, the set of human minds (psyches) created culture, science, fine arts, ethics and religion, which in turn had a great impact on formation of particular minds (psyches) themselves. For this reason, the evolution of the (collective) conceptual network of culture had to proceed in parallel with the evolution of civilization.

\subsection{Conceptual Network and Neural Network}

The conceptual network is an epiphenomenon (result of supervenience), aspect or by-product of the activity of the (properly dynamically organized) neural network in the brain. The functional unit of this network is constituted by a neural cell (neuron), which is functionally connected with other neurons, receptor cells and/or effector (motor) cells. Together, they form a (broadly understood) neural network. The activity of a single neuron within this network consists in perceiving stimuli (impulses) from other neurons or receptor cells through appendages called dendrites, processing these stimuli with the participation of information already possessed by a neuron (memory), and a possible (optional) transfer of a signal of an appropriate intensity (impulse frequency) to other neurons or effector (predominantly muscle) cells through an appendage called axon. The axon of one neuron is connected to dendrites or the cell bodies of other neurons through synapses. Only the whole complex of neurons, receptors and effectors forms a closed functional system, within which neural signals are transferred and which constitutes the material "base" of the conceptual network (Korzeniewski, 2013a, 2014, 2017).

The evolution of the conceptual network in the individual development (ontogenesis) of man constitutes an aspect or epiphenomenon of the evolution of the neural network underlying it. The latter consists of the formation of new neural connections (appendages and synapses), the decay of a part of the existing connections, the change of the "weight" (excitability) of existing synaptic connections, the biochemical record of memory, the origination of impulses circulating in neural circuits, the selection of existing circuits, and perhaps of other, yet unknown processes. All these processes occur in the brain, the general scheme of the structure and function of which is innate (encoded genetically).

Sensations as well as primary and secondary concepts are epiphenomena, aspects, or by-products of integrative structures as well as lower- and higher-level associative structures in the brain, respectively (Korzeniewski, 2010, 2017). Stimuli coming from sensory receptors constitute the primeval "substance" from which concepts are created. They are a certain form of activation of complexes of neurons with a characteristic spatiotemporal pattern. The structure of neural 
connections in a given part of the brain, for instance in the visual cortex (and also their communication via the visual nerve with photosensitive cells in the eye), ascribes a given complex of impulses to a visual sensation and not, for instance, to a hearing of sounds. The dynamic neural structures responsible for appropriate processing of stimuli from receptors are the so-called integrative structures (Korzeniewski, 2010, 2017). At least lower-level integrative structures are inborn. For instance, in the case of the sense of sight, the structure of connections in the visual cortex decides whether a given (broadly understood) "picture" has mostly a spatial character or whether it is focused on movement perception. Generally, the picture (representation) of the world created within the brain is by no means identical with, for instance, the spatial arrangement of stimulations of photoreceptor cells in the plane of the retina. What the brain sees is not what the eyes see. In turn, neural structures of a higher order, named lower-level associative structures (Korzeniewski, 2010, 2017), within which sensations are associated according to determined patterns, underlie concepts, namely primary concepts. Lower-level associative structures are created on the basis of incoming similar complexes of sensations that coincide in time (arrive at the same moment). They represent particular concrete objects, processes and events in the real world. The existing lower-level associative structures enable in turn interpretation and understanding of the incoming sensations, attributing them with meaning. Association on a still higher level is the source of higher-level associative structures underlying secondary concepts. They are created by association and consolidation of lower-level associative structures (and less abstract higher-level associative structures) as well as extraction of repeating patterns and structures in their complexes. This process is equivalent to broadly understood thinking and operational memory. Higher-level associative structures represent general laws, rules, ideas and universal beings. There is no sharp delimitation between lower-and higher-level associative structures and several sub-levels can be distinguished within them. Overall, the "substance" of all concepts is a derivative of sensations (integrated stimuli from receptors) and their processing (association) by "higher" centres of the brain. However, it should be stressed that sensations themselves constitute (an epiphenomenon or aspect of) sets of neural impulses received from receptors that have been integrated and associated by, partly inborn and partly acquired during individual development, neural mechanisms.

\subsection{Conceptual Network and Language}

The discrete, denotative language can be opposed to the continuous, connotative conceptual network (Korzeniewski, 2010, 2013b, 2014, 2015, 2017). Nevertheless, at a deeper level, language constitutes a part of this network, as it is built of (complexes of) concepts, which lie at the base of linguistic names, sentences and phrases as well as their meaning. Discrete, clearly-separated names in language correspond to best specified, most distinguished and univocal, endowed with 
most "intense" meaning concepts in the conceptual network. Misty, vague, poorly defined and difficult to interpret concepts do not have their counterparts in the domain of language. Therefore, the conceptual network is a phenomenon decidedly more general and more primeval than language. For this reason, just concepts and their complexes, and not linguistic names and sentences, constitute the content of human consciousness. Linguistic names mean nothing without concepts underlying them that constitute their "semantic lining". Language fulfils two main functions. The external function is communication between two or more psyches or conceptual networks constituting their substance. The internal function is a very efficient operation (manipulation) with individual concepts and the whole conceptual network, and thus enhancement of the very processes of thinking. This fact led to conceptions of "linguistic thinking" (Wittgenstein, 1922). Most probably, a highly developed symbolic language is necessary for origination of higher forms of thinking and (self-)consciousness. Nevertheless, this is concepts, that is, the fundamental "substance" of psychic processes. Undoubtedly, language has a huge impact on the representation of the reality in our brain and mind, as it strongly co-forms, but also immensely deforms this representation (Korzeniewski, 2013b).

The "fact-creating" role of language determines to a large extent the essence and structure of the conceptual network of, on the one hand, individual mind, psyche, and self-consciousness and, on the other hand, of the whole of human culture, including science, fine arts and literature (their subjective reception), philosophy, ethics, religion, and the sphere of common senses (Korzeniewski, 2013b, 2017). Language shapes the form and content of human thinking and directs its course. Science differs from, for instance, most of philosophy, humanities or religion by its methodology, which ensures congruence between linguistic (and conceptual) structures in subjective minds and inter-subjective science, and the structures of different aspects of the world. Nevertheless, this congruence is decidedly not perfect and this differs real science from its idealizations. There frequently takes place a naïve belief in a real and "sharp" existence of such objects described by science as the orbital of an electron, probability wave, gravitational force, or biological species, which constitute general concepts. The same also applies to a particular electron, the planet Earth and a given, concrete individual of bacterium or bat (Korzeniewski, 2013b, 2017). In this case, the qualification "real" does not mean an existence totally outside our psyche, which should by no means negated, but in the forms, categories, or even values produced by the human mind. At the same time, it is not true that Kant's "things in themselves" (noumena) are completely non-cognizable (Kant, 1999). If our categories, view of the world and the conceptual network underlying them had nothing in common with the structure of the real world, we would not survive as a biological species, and all the more we would not achieve such a spectacular success in natural sciences, technology and medicine.

According to philosophers that prefer analytical philosophy and linguistic thinking modo Wittgenstein (1922), a clear boundary separates the known from 
the not-known. The known constitutes the already ready part of our knowledge, grasped in linguistic structures and therefore true for ever, while the not-known is the entire rest, waiting to be pushed into the corset of linguistic structures. On the other hand, the conception of the conceptual network postulates that nothing can be absolutely true or false in our representation of the world (Korzeniewski, 2013b, 2014, 2017). Our cognition is a continuous process, which consists of the refining (or rather: co-refining) of concepts, and in co-development of apparently distant areas (maps) of the conceptual network that can present in a new light, or even change completely, these elements of our knowledge that we currently regard as unshaken and obvious, and also in elimination of the fragments of the conceptual network that no longer seem to represent adequately the external world. The process of cognition occurs gradually and never leads to completely certain, absolute, ultimate knowledge. Even logic (and also mathematics), which could seem to be an absolute and autonomous gauge of the unity of at least the most basic properties of our mind (language), on the one hand, and the objective reality, on the other hand, is nothing more but an evolutionary-grounded mechanism by which our brain treats stimuli from the environment (Korzeniewski, 2013b, 2014, 2017).

\subsection{Conceptual Network of Culture}

The collective conceptual network of culture is formed by individual conceptual networks of particular society members and, in turn, co-shapes these networks. The conceptual network of culture is in a sense a sum, an average and/or a derivative of individual conceptual networks of its participants. As such, it is less determined and more vague ("blurred") than the latter. Culture constitutes a form of collective communication of people, both in space and in time. It binds sets of individuals (in particular: individual conceptual networks) into complicated "organisms" of societies having a complex, hierarchical structure and multiple internal relations.

The culture as a whole is related to the conceptual networks of people living in it and creating it. In this context, the psychological aspect of culture, and not, for instance, its material products, is of special importance. It can be said that the conceptual network of culture constitutes something like the Popper's third world comprising a complex of common believes, religion, ethnical language, scientific knowledge, ethical and aesthetic values, masterpieces of fine arts and literature (in their inter-subjective reception), arbitrary conventions and so forth, situated beyond the material world (the first world) and human minds (the second world) (Popper, 1978). Culture (in particular: the conceptual network of culture) is attributed with autonomous existence, not reducible to psyches (conceptual networks) of individuals participating in it. The conceptual network of culture is not a separate and "absolute" philosophical being. Rather, it is a convenient conventional category, a commonly understood concept accepted because of its usefulness in ordering, describing, and interpreting the 
world of phenomena accessible to us (Korzeniewski, 2017). Of course, the conceptual (sub-)network of science constitutes a component of the collective conceptual network of culture.

The amount of information contained in the conceptual network of culture exceeds greatly the capacity of the human brain, of single human conceptual network. For this reason, the former can exist only outside any consciousness, in the domain of abstract beings. Additionally, there are no two identical individual conceptual networks. Therefore, the conceptual network of culture must be composed of more vague concepts than the concepts entering into the composition of individual conceptual networks, in which the "cultural" concepts are specified for given persons' own use (Korzeniewski, 2017).

\subsection{External World, Neural and Conceptual Network, Language, and (Self-)Consciousness}

The continuous in its essence external reality (Korzeniewski, 2013b), its aspects, objects, processes and "facts" are represented by primary and secondary associative structured in the neural network of the human brain. This network can be also regarded as continuous, although it is composed of discrete neurons equipped with discrete appendages (axons and dendrites) and synapses. First, the frequency of neural impulses conducted by the axon, amount of neurotransmitter released to the synaptic cleft and the synapse excitation threshold can change in the continuous manner. Second, thousands of neurons underlie particular concepts, and therefore single neurons are below the "resolution" of mental processes, as pixels in a good photograph are below the resolution of our sight. Thus, the continuous conceptual network composed of meaning by connotation concepts that smoothly pass one into another is and emergent property (effect of supervenience) of the appropriately-organized activity of continuous in the macroscopic scale neural network, composed of neurons and their complexes that also mean by connotation. The conceptual network constitutes the substance of the human psyche and mental objects contained in it, which are also represented as concepts within the conceptual network.

It was postulated that self-consciousness emerges from the neural network, when this network in the cognitive centre in the brain (located in the prefrontal cortex, the seat of the operational memory of the brain) becomes recurrently directed on itself, begins to receive neural signals from itself (the cognitive centre of an unconscious brain receives only signals from receptors, processed and integrated by sensory cortex) (Korzeniewski 2010, 2013a, 2015, 2017). As a result, the conceptual network underlaid by the neural network also becomes directed on itself, creates its own representation (model) within itself, refers (on a certain semantic meta-level) recurrently to itself, perceives its own existence. The "true", psychic consciousness, as opposed to instrumental consciousness equivalent to representation of some object within a certain subject (e.g., of mobile picture within a video camera or a fly within the frog brain neural network), cannot exist 
without self-consciousness (Korzeniewski, 2010, 2017). Finally, discrete names of language correspond to best determined, separated and specified concepts (and their complexes) endowed with the most intense meaning. Linguistic names are attributed to concepts representing "facts" of the external world, mental objects and the very (self-)consciousness. However, they do not denotate directly the things they refer to. Just the opposite, they do this only indirectly and approximately, by mediation of the conceptual layer (Korzeniewski 2010, 2013a, 2015, 2017). The relation between noumena of the external reality, concepts in the conceptual network (underlaid by neuron complexes in the neural network), mental objects, linguistic layer and self-consciousness are presented schematically in Figure 1.

\subsection{Rationalism vs. Empiricism}

General properties of the structure and function of the human brain, neurons

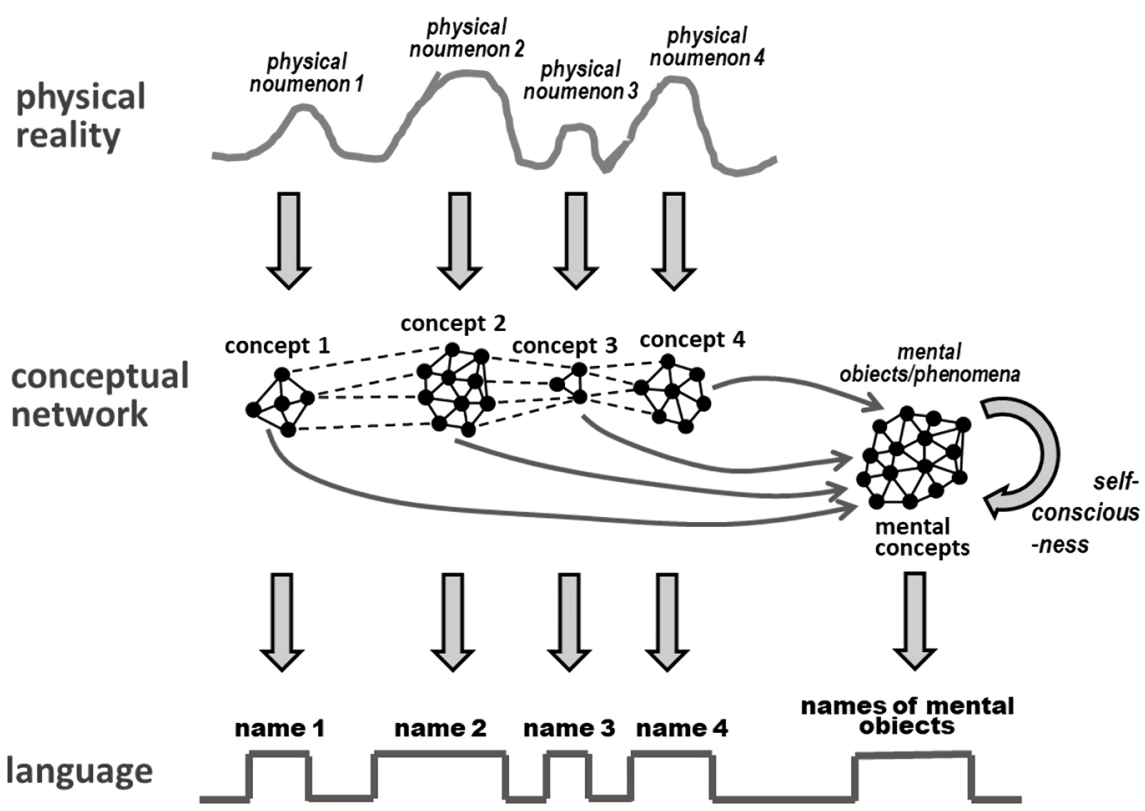

Figure 1. Relation between physical reality, conceptual network, mental phenomena, language and self-consciousness. The noumena (aspects, objects, processes, "facts") of the continuous in its essence physical reality are represented as meaning by connotation concepts within the continuous conceptual network that constitutes the substance of psyche and mental objects contained in it. The conceptual network is underlaid by the also continuous, in the macroscopic scale, neural network (not shown). Also mental objects are represented by concepts within the conceptual network. Self-consciousness (and thus psychic consciousness) appears when a part of the conceptual network becomes recurrently directed on the (potentially) entire network, forms its own representation (model) within itself (the neural network in the cognitive centre in the brain underlying it starts to receive signals from itself, perceive its own content and activity). Discrete linguistic names correspond to best determined and separated concepts, endowed with most intense meaning, that correspond to different aspects of the material world, various mental objects and processes as well as to the phenomenon of self-consciousness, feeling of one’s own ego, "I". 
and neural networks responsible for integration of sensory stimuli, for instance into spatial, temporal and causal structures (lower-level integrative structures), as well as the overall manner of higher-order data processing (including the general form of thinking processes) are innate, determined by the genetic record. They co-shape our representation of the world and processes that lead to its formation. This is the "rationalistic" contribution to our cognitive activity. However, particular synaptic connections and their excitation thresholds, detailed functional structure of most of the neural network, semantic, episodic and procedural memory records as well as particular modes of higher-level data processing (particular thinking processes) (lower- and higher-level associative structures) are acquired during individual development (life). This is the "empirical" component of our cognition (Korzeniewski, 2017). The "rational" is "subjective", while the "empirical" is "objective", although not in the naïve sense, as the representations of various aspects of the external reality in human minds are far from identical with these aspects themselves. Even the structure of the neural and conceptual network acquired during the individual development is co-shaped by inborn neurophysiological mechanisms of signal integration and association. Therefore, there is no sharp borderline between "rational" and "empirical", and they overlap to a significant extent. The "rational" thinking is co-created by individual experience, while the "empirical" knowledge and its formation is partly underlaid by inborn neural mechanisms. On the other hand, the inborn "rational" functional structure of the brain, neurons and neural networks is in a sense also "empirical", as it has been formed ("learned") in the course of biological evolution. Only such kinds of the general architecture and functioning of the neural systems have passed the sieve of the natural selection, which allowed to increase the fitness of organisms "equipped" with them. Therefore, the general form of these networks had to represent more or less the external (physical, biological and social) reality, or at least these of its aspects that were important for natural selection (survival and reproduction). On the other hand, we ought to remember that the brains of our ancestors evolved to hunt for mammoths, and not to develop science and philosophy.

\subsection{Phenomena vs. Noumena}

The relation of the human knowledge, picture of the world in the human mind and the representation of the reality in human senses (phenomena), in particular of scientific theories, to (various aspects of) the world itself (noumena) was considered by numerous philosophes dealing with epistemology, philosophy of cognition and philosophy of science. Most of them regarded that either our mental pictures represent well some aspects of the world (primary ideas in Locke's terminology, e.g., shapes; Locke, 1690) and much worse other aspects of the world (secondary ideas in Locke's terminology, e.g., colours; Locke, 1690), or they have in fact very little to do with the on principle unknowable "things-in-themselves" (noumena) (Kant, 1999). Concepts formed on the basis of induction, such as the 
concept of "cause", have no absolute justification and can represent the real world only imperfectly and approximately (Hume, 1975). According to Kant (1999), all categories in which we perceive the world (such as space, time, causality, particular objects, shapes, distances etc.) are created by human mind (psyche) and characterize (are aspects of) mental objects (phenomena), but do not exist in the external world of noumena. We have by definition no access to, and can gain no knowledge about the latter.

The philosophy of conceptual network (Korzeniewski, 2010, 2014, 2017) says that humans possess within the conceptual network a certain, although imperfect, representation of the noumena of the external world. While the substance of the conceptual network is completely different from (has a completely different nature, than) the substance of the physical reality, then the structure of the former adheres to some extent to (is somehow isomorphic with) the structure of the latter. Therefore, the external world is only partly, but not entirely (un)knowable by the human mind.

\section{Conceptual Network and Philosophy of Science}

\subsection{Conceptual Maps of Scientific Theories and Their Evolution}

The conceptual network of science is a special fragment of the overall conceptual network constituting the content of the human psyche and underlain by the cognitive neural network in the human brain. The individual, personal conceptual network of science appearing within the brains of particular scientists and underlaid by their neural network in general, as well as the conceptual maps and sub-maps of its disciplines, sub-disciplines and theories, exhibit all the features that characterize the conceptual network as a whole. Concepts and complexes of concepts within it mean by connotation. The network develops by splitting of old concepts into new concepts, adding new concepts on the basis of the existing network, further determination of the already existing concepts and elimination of the concepts that do not fit to the evolving structure of the network, including those of its fragments that are formed on the basis of newly incoming sensations. New conceptual maps and sub-maps of scientific theories and hypotheses can be created on the basis of the existing conceptual network of a given scientific discipline (or several disciplines in the case of interdisciplinary science branches). The primary (empirical) source of new concepts (especially primary concepts) are incoming sensations, that is external stimuli received by sense organs and processed by integrative neural structures in the brain. These stimuli and sensations can constitute elements of observations or experiments. However, their interpretation and understanding is conditioned by the existing structure of the conceptual network of science. The secondary (rationalistic) source of new concepts (especially secondary concepts) and the mechanism of the development of the conceptual network of science is an autonomous activity of the conceptual network and the neural network underlying it. These are broadly understood thinking processes consisting in the functioning of higher-order associative 
structures and formation of new associative structures on the basis of the existing ones in the brain. They underlie secondary concepts. The crucial characteristics of our world view in general, and of scientific theories in particular, is the connotative character of neurons and associative structures, on the one hand, and of concepts and their complexes, on the other hand.

The collective, inter-subjective conceptual network of science understood as a social phenomenon can be regarded as a part (sub-network) of the conceptual network of broadly-understood culture (Korzeniewski, 2017). Culture in general has a predominant impact on the origin and development of science (Weber, 2001). A perfect translation of two ethnic languages used in two cultures into each other is impossible (the so-called indeterminacy of translation; Quine, 1960). In particular, the structure of the ethnical language of a given culture determines to a significant extent the structure of cognition and view of the world of the participants of this culture (Sapir, 1921; Whorf, 1940). This statement concerns also the structure of science developing within a given culture and language. Because of cultural and linguistic determinants and limitations, the development of science can stop at a very early stage, as it had happened in most cultures that ever originated on the planet earth. Nevertheless, if under circumstances of a given culture and its language science is successful to develop, it must represent better or worse the external world because of its very nature, especially methodology. As, this methodology ensures a current (or with a finite delay) testing of the degree of adherence of the conceptual network of science and its (sub-)maps (theories) to the physical reality.

Creation of scientific theories occurs initially within the individual conceptual networks of particular scientists, while their common acceptance or rejection within the collective conceptual network of science. The latter is of course a derivative of the individual networks of the scientific society participants, but at the same time co-shapes reciprocally these networks. Therefore, the mutual interactions between individual conceptual networks of particular scientists and the collective conceptual network of science are substantial for the nature and development of science. Invention of novel theories (scientific conceptual maps) constitutes a result of the autonomous activity (equivalent to thinking processes) of the individual conceptual network(s), and the neural network(s) underlying it (them). The confrontation of conceptual maps of new scientific theories and hypotheses with the existing conceptual maps of theories, observations and experiments is carried out by individual scientists and teams of scientists, and its results belong to the collective conceptual network of science. Similarly, the conceptual maps of new theories are being referred to new sensations related to new observations and experiments, but also to various existing, well-established theories, and the whole scientific society decides whether the former fit satisfactorily to the latter or not. In the first case the reliability of new theories is strengthened, while in the second case the trust in them is weakened or they are simply abolished (rejected). The conceptual maps of new consolidated theories 
undergo location within the "collective scientific memory records", that is in the collective conceptual network of science (its appropriate discipline). Overall, the mutual bi-directional interaction between individual conceptual networks of particular scientists and the collective conceptual network of science leads to analysis and comparison of various conceptual maps, extraction of repetitive patterns in different complexes of secondary concepts (higher-order associative structures), formation of a new, differently-structured conceptual maps (theories), relating them to the already existing conceptual maps as well as known and newly collected facts (observations and experiments). Such differently-structured conceptual maps of new theories are able to infer both the conceptual and "real" structures of new, still undiscovered aspects, objects and processes of the physical world, and thus predict their existence. This, in turn, results in constructing conceptual sub-maps related to planned future observations and experiments. Initially, all this happens within the individual conceptual networks of particular researches. Next, if the new conceptual map has passed through self-criticism of its inventor(s), it is presented to the scientific society. Other scientists analyse its logical, mathematical and conceptual structure, confront it with the presently known (through observations and experiments) properties of the real world, refer them to well-established (conceptual maps of) theories from a given scientific field and related disciplines, estimate its heuristic fruitfulness as well as plan and eventually carry out observations and experiments that could test (support or abolish) this theory (its conceptual map). If the decision about the preliminary acceptance of the theory by at least a part of scientists is positive, the theory becomes consolidated in the collective conceptual network of a given branch of science. Particular scientists further determine, specify and interpret new theories for they own use, relate them to their individual conceptual networks. Therefore, there are in fact no two identical representations of a given scientific theory within individual conceptual networks of particular researchers. Next, a validity of a preliminarily-accepted theory is further tested by subsequent observations, experiments and theoretical analyses. Finally, the analysed theory ceases to be able to account for new observations and facts, explain other aspects of reality than those it hitherto described properly, and be reconciled with other, well-established theories (its conceptual map is not congruent with their conceptual maps). In such a situation, a new, more fundamental theory is awaiting formulation, that would reach to an even deeper layer of the reality and unify the exiting theories that hitherto fell into contradictions. At present, for instance quantum mechanics and general relativity are such theories that are awaiting unification on a certain deeper level, and the string theory and loop gravity are most frequently discussed candidates for the unification theory (see e.g., Greene, 2005; Penrose, 2002). A separate problem is whether the cognitive capabilities of the human brain and mind are efficient, vast and universal enough to create such a general theory unifying entire physics, at least on the dynamic level. Most probably, e.g., thermodynamics cannot be reconciled with dynamics, as these 
two scientific paradigms operate with completely irreconcilable concepts and logics. Generally, the conceptual apparatus of the classical (dynamic) physics does not allow it to speak about the phenomenon of complexity that seems to me to exists as objectively, as space, time, matter and other purely "physical" phenomena and is related to the phenomena of life and (self-)consciousness (Korzeniewski, 2015). Therefore, the very language and logical structure of dynamics cannot speak about such systems as thermodynamic systems, complex chemical systems, living individuals and conscious beings (Korzeniewski, 2010, 2013a, 2017).

One more fact becomes evident within the framework of the philosophy of science based on the philosophy of conceptual network. When a given old theory that hitherto worked well for a long time and was able to account for a broad spectrum of various phenomena is ultimately rejected by the scientific society (within its collective conceptual network of science) as not good and universal enough, contradicting new observations and experiments and not being sufficiently consistent internally and externally (with other theories), it is by no means falsified. It is only shown that the theory has certain limitations and should be replaced by a new, more advanced theory that adheres better to the structure of a greater number of various aspects of the external world. Nevertheless, the old theory still represents well many aspects of the reality, the structure of its conceptual map adheres fairly well the structure of the world. An ultimate falsification of any theory would be possible only if the aim of scientific theories, and of science in general, were the ultimate and absolute truth. This is decidedly not the case, at least in the frame of the philosophy of conceptual network (Korzeniewski, 2014). Therefore, no real falsification of hitherto well-working theories, at least in relation to some spectrum of phenomena, can take place. The conceptual map of the new theory that is intended to represent better a given fragment of the reality, developing in a sense de novo from some conceptual germ, has an essentially different structure (better adhering to vaster areas of the reality), than the conceptual map of the old theory, with different concepts and connotative relations between them. Nevertheless, these maps (some fragments of them) must also adhere somehow to each other, at least partly, as they refer (adhere to some extent) to the external world. The Newtonian and Einsteinian gravity theories can serve as an example. Although Einsteinian general relativity better adheres to the structure of the physical world, Newtonian dynamics is still doing well and has been by no means falsified!

This paragraph describes the core of what I would like to call the Conceptual-Network-Based Philosophy of Science (CNBPS). This conception will be developed, specified and further discussed in following sections, among others through referring it to the existing philosophies of science.

\subsection{Existing Philosophies of Science vs. Conceptual Network Philosophy}

Inductionism (Salmon, 1975; Russell, 1912) is probably the simplest and most 
natural, and historically first, theory of the science development. According to it, science and scientific theories are based on laboriously collected observations that are primary in relation to theories. The theory is the more reliable, the greater number of observations it is based on. In the light of inductionism scientific theories are in fact common features of observations extracted from them and a little refined. Inductionism was criticized from many points of view. The most important is the argument that observations are not independent autonomous acts, but they strongly depend on theories and knowledge of the observer. In real science, theories usually precede observations (observed "facts" of the world, observation statements). Observation statements are equally abolishable as theories. Every observation statement is formulated in the language (terms) of some theory. There takes place a reciprocal, bi-directional relationship between observations (as well as experiments) and theories. Theories usually appear before observations and experiments necessary for their validation are made, and determine their character. Inductionism does not take into account the enormous complexity of the process of scientific cognition (Chalmers, 1999) and the relational nature of scientific meanings, and ignores the fundamental matter of how scientific theories originate in the brains of scientists.

According to conceptual network philosophy, new sensations (processed and integrated stimuli received by receptors from the external world) can be interpreted, understood and eventually inbuilt into the conceptual network only on the basis of the already existing conceptual network. Using terminology closer to science, new observations are not assimilated "in void", by a "pure reason". They are located within the already existing knowledge and scientific theories, related to and interpreted by them. Incoming sensations can be potentially inbuilt into the structure (conceptual network) of the knowledge/theories and contribute to their development. Therefore, the relation here is bi-directional and in fact connotative: new observations mean in the context of current theories (their conceptual structure), while these theories can be modified and developed in a sense de novo from some conceptual germs by accumulation of new observations (and complexes of concepts underlying them) and their integration and processing (thinking processes, operating with associative structures). This conclusion concerns to an even higher degree experiments that must be planned and carried out on the basis of the current scientific knowledge and theories.

Falsificationism (Popper, 1968) seems to constitute a certain progress in relation to inductionism, especially the naïve one, but it also has serious limitations and encounters numerous problems. The act of falsification can be falsified as well, as it depends on theory. Of course, any scientific theory must be falsifiable, and the more falsifiable a theory, the better it is. This is a necessary, but by no means sufficient condition for a theory to be scientific. Additionally, a given theory can be rescued from immediate falsification by formulation of ad hoc hypotheses, that sometimes appear to be correct, as it has happened in the case of the discovery of planet Neptune. Falsificationism usually does not take into 
account the complexity of each experimental situation. It says little about how scientific theories are created and justified. It does not reflect the relational nature of scientific meanings. Finally, and perhaps most important, when it is ultimately (at least according to the current knowledge) demonstrated that a given theory, that hitherto worked well for a long time and was able to account for a broad spectrum of various phenomena, contradicts some (new and already known) observations or results of experiments, this theory is by no means falsified. It is only shown that the theory has certain limitations and should be replaced by a new, more advanced theory that adheres better to the structure of the external world. Nevertheless, the old theory still represents well many aspects of the reality. An ultimate falsification of any theory would be possible only if the aim of scientific theories, and of science in general, were the ultimate and absolute truth. As this is nonsense, no real falsification of well working theories, at least in relation to some spectrum of phenomena, can take place. As mentioned above, a good example is the Newtonian gravitation theory that has been replaced by Einsteinian general relativity (a more advanced, but certainly not explaining everything and ultimate theory), but not falsified (it still remains a very good approximation of many aspects of the reality under many conditions, for instance at speeds much less from the speed of light, and its structure adheres well to the structure of a large fragment of reality).

From the point of view of the conceptual network philosophy, "falsification" of a theory (or, rather, demonstration of its insufficiency) takes place when its conceptual map fails to adhere to the conceptual maps of new observations and experiments (and also of some old observations, experiments and well-established related theories from neighbouring fields that cannot be grasped by the conceptual apparatus of this theory), is not able to accommodate them as a part of its own conceptual map. However, one should bear in mind that both the tested theory and the new experiments or observations can be "false", that is the structure of their conceptual maps can be incoherent with the structure of an appropriate aspect of the reality. Anyway, even if new observations and/or experiments are proven to be "true" (to represent adequately some aspects of the real world), the old theory that they contradict is not falsified, but only shown to have a limited applicability, as its conceptual map still fits well to the conceptual maps of old observations and experiments it was hitherto able to describe correctly. The internal connotative relations between the complexes of concepts underlying this theory are still at least approximately isomorphic with some "facts" of the physical world.

According to Kuhn (1962) science works for most of the time as "normal science", exploiting and exploring the capabilities of a given scientific paradigm. When this process falls into a serious crisis, when more and more observations and experiments cannot be reconciled with the current paradigm, a revolution takes place and a new paradigm appears that is differently internally structured, than the old paradigm. If this new paradigm appears to be temporarily success- 
ful, science (a given branch of it) passes to a new period of "normal science", until a new crisis follows and a new, more appropriate paradigm emerges. In Kuhn's opinion, sociological factors play a crucial role in the appearance and acceptance of new scientific paradigms and the development of science heads no external or objective truth (relativistic position) (Kuhn, 1962).

In a sense a more elaborated version of the Kuhn's philosophy of science was formulated by Lakatos (1970). Here, for most of the time science realizes (follows) particular "research programmes". They are composed of a hard core containing fundamental and unshakable assumptions (defining properties of a given programme, theories with a determined structure) and a protective belt that protects the research programme against falsification. This belt contains auxiliary ad hoc hypotheses, initial conditions, methodological assumptions (also concerning observations and experiments), et cetera. Modifications and supplementations of the protective belt cannot be arbitrary, but must be independently testable. Research programmes can be progressive or degenerative. Progressive programmes are still successful in explaining new phenomena, while degenerative programmes are not heuristically fruitful any more and fall into contradiction with an increasing number of observations and experiments. As a consequence, an old degenerative research programme is in a certain moment replaced by a new, at least temporarily progressive research programme, with a new hard core and fundamental structure, until it will begin to degenerate. Then, a given scientific field is taken over by an even more advanced research programme, the fundamental structure of which adheres even better to an even larger area of the reality. Lakatos believed in a real progress in science (rationalist position) (Lakatos 1970).

Overall, the philosophy of conceptual network conforms pretty well with the philosophies of science by Kuhn and Lakatos. The emergence of a new scientific paradigm (Kuhn, 1962) or research programme (Lakatos, 1070) is related to formation of a new conceptual map (a relatively well-separated and internally consistent fragment of the conceptual network; Korzeniewski, 2014) within the conceptual network of science on the basis of the already existing conceptual network as well as new observations and experiments leading to creation new concept complexes. A new paradigm or scientific programme, corresponding to a new theory or set of related theories, usually has a different internal conceptual structure than the old, abandoned paradigm or scientific programme. Similarly, a new conceptual map that is intended to represent better a given aspect of the reality, developing in a sense de novo from some conceptual germ, has an essentially different structure (better adhering to vaster areas of the reality), than the old conceptual map, with different concepts and connotative relations between them. Nevertheless, these (old and new) maps (some fragments of them) must also adhere somehow to each other, at least partly, as they refer (adhere to some extent) to the same external world. As mentioned above, new conceptual maps (theories) are created first within individual conceptual networks of particular re- 
searches. The autonomous activity of the neural network (thinking processes) that consists in creation, modification, development and elimination of higher-order associative structures (secondary concepts) underlies this process. Eventually, such "private" conceptual maps are preliminarily transferred to the inter-subjective (inter-personal) conceptual network of science. Then they are estimated by the scientific society (individual conceptual maps of its members) and can be accepted, preliminarily (conditionally) accepted or rejected. The new scientific conceptual maps (theories) must to be able to account for the features of the physical world, which were well explained and described by old maps (theories) and at the same time to represent correctly new phenomena, not grasped by old maps. The new conceptual maps are also expected to be more internally consistent, than the old maps, to operate with better specified, more precise concepts with a denser network of connotative relations between them. Nevertheless, new scientific theories are frequently created within the minds (conceptual networks) of scientists before they can be verified by new observations and experiments. Only after such a successful verification a given conceptual map (theory) representing a certain aspect of the physical world (Popper's first world) can be definitely transferred from the personal subjective conceptual network of a researcher (Popper's second world) to the inter-personal (inter-subjective) conceptual network of science (Popper's third world) and consolidated in the last.

A scientific theory (its conceptual map) is good if its structure adheres well to the structure of a broad range of various aspects of external reality and if it is based on methodology (experiments, observation, mathematical models, validation) than allows to estimate and improve the relative degree of this adherence. The research programme is progressive (in the Lakatos's sense; Lakatos, 1970), if it offers a realistic way to increase the degree of the adherence of the conceptual map of the theories constituting its core to the reality and to enlarge the number of the aspects of reality to which it adheres. In other words, the structure of the conceptual network of a good scientific theory should represent well (adhere to) the structure of various aspects/facts/phenomena of the real world. A perspective theory should be able to tighten the meshes of its conceptual network around the "knobs" ("protrusions") of the external reality well represented up to now by this theory (and previous theories), and to represent new aspects of this reality by creating and developing new knots of this network.

Representative realism (Locke, 1690) says that our concepts corresponding to some aspects of the external world are completely dissimilar to those aspects (Locke's secondary qualities). Unrepresentative realism (Chalmers, 1999) says that scientific theories apply to different extents to the real world, but they do not approach the truth in any understanding. Therefore, this conception seems a little instrumentalist. The philosophy of science based on the philosophy of conceptual network (Korzeniewski, 2014) can be classified as semi-representative realism. It says that the (complexes of) concepts representing some aspects of the external world are of completely different nature, than those aspects themselves. 
In other words, the "substance" of the conceptual network is irreconcilable with the "substance" of the reality. On the other hand, the structure of (complexes of) concepts corresponds to a certain extent to (is to some degree isomorphic with) the structure of the aspects of the world they represent. These structures adhere, better or worse, to each other. Therefore, science aims to approach somehow the truth, although in an imperfect, approximate, relative and relational way. Scientific theories and their conceptual maps have no chances to reach the absolute, ultimate truth. The adherence of the structure of conceptual maps of scientific theories to the structure of the physical reality is gradable, but never prefect. In other words, scientific theories can be more or less true, but never absolutely true. Scientific, especially physical theories do not reach to the very heart of the essence of the external reality. They only formulate (mostly mathematical) descriptions and prescriptions that allow to represent possibly faithfully a possibly large number of various physical phenomena and are able to predict successfully a possibly broad spectrum of new phenomena. As such, they say nothing about the very heart of the nature of the physical world. They do not offer (and they will never do) an absolute and ultimate Truth.

As mentioned above, the conception presented in this work can be called the Conceptual-Network-Based Philosophy of Science (CNBPS). According to CNBPS the development of science consists in the development of the conceptual network of science: increase in its size (increase in the number of concepts); increase in its "resolution" (further determination and specification of the existing concepts and conceptual maps); increase in its congruence (adherence) to various aspects, facts and phenomena of the real world; origination of entire new conceptual maps and sub-maps (theories, paradigms, research programmes).

CNBPS stands on a position between extreme rationalism and extreme relativism. It postulates that a long-term development of science is to a large degree independent on sociological factors, as the structure of science must adhere, better or worse, to the structure, unique and univocal in its essence, of the physical world. This is ensured by the methodology of science. On the other hand, CNBPS rejects the opinion that the role and ultimate aim of science is to reach some absolute truth, as this is on principle impossible (Korzeniewski, 2014). The development of science should be directed to an increase of the degree of the congruence of the structure of conceptual (sub-)maps of scientific theories to the structure of various aspects of the external reality that exists objectively "out there", without any hope that we will ever know the very essence of its nature. Nothing more and nothing less. Even if in a given culture science starts from "wrong" departure points, it can always change the current paradigm, replace old research programme with a new one and develop a new conceptual map of science, as long as it complies with scientific methodology. Otherwise, the development of science will die out at a very early stage, as it has happened in most cultures that originated on our planet.

CNBPS is decidedly more objectivistic than individualistic (Chalmers, 1999). 
It assumes the objective existence of the external reality, which is however only to some extent cognizable for the human mind. The role of science is a possibly good adherence of the conceptual maps of science to possibly large areas (numerous aspects) of the real world. On the other hand, CNBPS does not deny that science is a result of human activity (or alien activity on other planets in the universe), can develop to some extent accidentally, by a chance, and be co-shaped by the particular culture and ethnical language it develops within. It is by no means going to reach some absolute and ultimate truth about the world.

CNBPS is also located between realism and instrumentalism, although closer to the former. It says that scientific theories (their conceptual maps) describe, represent the real world existing objectively "out there". In other words, the structure of the conceptual maps of scientific theories is to a certain degree isomorphic with the structure of the external reality. This is the "realistic" component of CNBPS. However, this description/representation is only approximate, as the conceptual maps of scientific theories adhere only imperfectly to the reality. What is even more important, scientific theories do not even try (regardless the intentions of their inventors) to reach to the very essence of the nature of external phenomena, but only aim to reproduce possibly well their formal structure (preferably with an aid of formal sciences, especially mathematics). The "substance" of the conceptual network of science is of completely different nature than the "substance" of the real world. This is the "instrumentalist" component of CNBPS. Nevertheless, the philosophy of sciences formulated within the philosophy of conceptual network does not share the extreme cognitive pessimism of Immanuel Kant (Kant, 1999) that "things-in-themselves" (noumena) are completely not cognizable. The position presented in this work can be called the semi-representative realism, in opposition to both representative realism and unrepresentative realism (Chalmers, 1999).

The advantages of CMBPS are as follows. It emphasizes the fact that concepts (senses) in the whole conceptual network in general as well as in the conceptual network of science and conceptual maps of scientific theories mean by connotation, by relation to each other (relational theory of meaning). It states that linguistic names refer directly to (complexes of) concepts, and not to aspects, objects and processes in the external world (they do not directly denotate them). It allows to analyse both individual conceptual networks in the brains and minds of particular researches, where scientific theories are born, modified and developed, and the collective conceptual network of science, where particular theories can be tested, accepted (at least temporarily) or rejected. It offers a description of the process of ideas transfer between individual and collective conceptual networks of science. It proposes how the conceptual network of science evolves, both in the periods of "normal science" working within particular research programmes, or during scientific revolutions, when new paradigms, research programmes and theories (conceptual maps) are created and replace old paradigms, research programmes and theories (conceptual maps). It says that hitherto well 
working for some time theories cannot be definitely falsified, but only shown to have limitations and replaced by more adequate, better adhering to the reality theories with their own limitations. It proposes that the progress in science consists in an improvement of the adherence of the structure of the conceptual network of science in general and conceptual maps of particular scientific theories in particular to the structure of the external reality. It postulates that an adequate theory of science should be situated somewhere between extreme rationalism and extreme empiricism, extreme rationalism and extreme relativism, objectivism and individualism as well as realism and instrumentalism. Finally, it demonstrates explicitly that science cannot on principle reach (or even tend to) some absolute and ultimate truth, as the structure of the conceptual network can only imperfectly adhere to the structure of some aspects of the world, and the very "substance" of the former is of completely different nature, than the "building material" of the latter.

The terms "truth" and "untruth" should be replaced within science (and everywhere else; Korzeniewski, 2014) by the term "relative degree of adherence to reality". The "truth value" of a scientific theory can be somewhere between 0 and 1 , but never exactly 0 or 1 . Tarski proposed the correspondence theory of truth (Tarski, 1935). Many thinkers regard this theory as trivial. However, it is in fact meaningless because of the vagueness of continuous concepts corresponding to discrete linguistic names (Korzeniewski, 2013b). Therefore, the statement " $\mathrm{X}$ is blue" or "Y is a table" can be only approximately, but not absolutely true or false. The same concerns, of course, all scientific theories and statements.

\section{General Discussion}

The most fundamental problem is whether science, or human cognition in general, aims to discover the absolute, ultimate truth about the universe, or whether it just tries to offer a possible adequate, exact, deep and universal description of the phenomena accessible to us, of measurable properties of the physical reality. At the same time, I do not mean what scientists or common people subjectively intend to do, but what they really do. In my opinion, the reaching to the very heart of the essence of the external (and internal) reality is impossible on principle, a priori. The conceptual network entwines the facts, objects, processes and aspects of the real world just as the spider web entwines a stony sculpture. The substance of the web is completely different from the substance of the sculpture, its adherence to the surface of the stone is only limited and the nodes of the web are not arranged densely enough to represent every detail of the sculpture surface. Similarly, the "substance" of the conceptual network, emerging from (being a result of supervenience of) the dynamic structure of the neural network in the human brain, is completely incomparable to the "substance" of the world. It represents only imperfectly and approximately certain aspects of the reality, and the density of its nodes (concepts) is not sufficient to reproduce the immeasurable variety of various properties and aspects of the world. Therefore, science can 
at best increase the resolution of the conceptual network (density of its knots concepts), elevate its degree of adherence to the external reality and extend the conceptual network to new aspects (areas) of the reality. The absolute, ultimate truth about the material (but also mental) world is out of the reach of science, and of human cognition and reason in general. Of course, while the spider web is in principle two-dimensional, the conceptual network is characterized by a huge (potentially unlimited) number of (semantic) dimensions (significative axes).

Generally, Conceptual-Network-Based Philosophy of Science (CNBPS) implies an interactive and relational (connotative) way (conception) of science and scientific theories development.

Theories that represent relatively well at least some aspects of the reality and worked relatively successfully for a certain time cannot be falsified, what was postulated by Popper (1968) and his followers. It is only possible to determine the limits of their applicability and degree of their adherence to the external world. Old theories that are replaced by new theories are not (entirely) false and the new theories that replace old theories are not (entirely) true. It can be said that the former are more false and the latter are more true, that is they adhere better to the "facts" of the world (noumena). The relative degree of this adherence in different theories can be estimated (compared) by the number of various aspects of the reality they are able to appropriately account for (and the number of aspects they cannot), which ensures a real progress in science. The quantitative exactness of the correspondence between a theory and relevant observations and experiments also counts in this context. The logical value of a given scientific theory is located somewhere between 0 and 1 , closer to 0 for older theories and closer to 1 in the case of newer theories. Nevertheless, it never equals exactly 0 or 1 , theories are never absolutely false or true (compare Korzeniewski, 2014). The Galilean and Newtonian dynamics or Maxwellian electromagnetic theory are not "false" (or fully falsified) for the reason that they have been replaced by the Einsteinian special and general relativity theory as well as by quantum mechanics, respectively. Similarly, the two last theories are not "true" (or ultimately verified), although they are able to account for a huge number of various physical phenomena. They simply better adhere to (determined aspects of) the physical reality (in macro- and micro-scale, respectively), than previous theories. Nevertheless, this adherence is still by no means perfect. Moreover, general relativity and quantum mechanics (their mathematical formalisms and their interpretations) cannot be reconciled, as they contradict each other. Most probably they should be unified on a more fundamental level and replaced by a new theory that would describe a deeper level of reality. On the other hand, it is not certain that this task is possible to be realized by the human brain and mind, by the conceptual network of science created by human civilization.

The sociological aspects of the Kuhn's theory of the development of science (Kuhn, 1962) are of minor importance, as the methodology of science ensures 
that the structure of a well-established scientific theory adheres, better or worse, to the structure of (some aspects of) the world. Sociological factors can temporarily (or ultimately in a given culture) suppress or delay the development of sciences, as it has happened in the middle ages in Europe, make it go astray for some time (as in communist Soviet Union or Nazi Germany), but they can determine the way and direction of its long-term evolution only to a limited extent. Sciences that emerge completely independently in different cultures would have to adhere to some extent to the structure of the external reality, and thus to each other. Alternatively, their development would stop in the embryonic stage. The ancient European (Greek, Roman), medieval Arabic and modern Western sciences can serve as partial examples of the convergent evolution of science, although they did not develop in complete isolation. Therefore, their (mathematical and conceptual) structures had to adhere more or less closely to each other. This statement concerns also "alternative" sciences developed by aliens on other planets in the universe.

As language is a universal tool that greatly increases our ability to use and operate with concepts (and thus enhances the development of the conceptual network), so science is currently the main generator of new concepts having a significant and deep correspondence with reality. New concepts emerge as a result of separation from the existing concepts through creation of new semantic relations between them, so far non-existent (as we remember, concepts mean by connotation, relation to each other). The "parent" concepts are also reciprocally modified as a result of interactions with newly formed "progeny" concepts they become better specified. The process of creating of new concepts is associated with diversification of meanings on the semantic axes as well as with origination of new axes. Of course, new concepts emerge in the whole area of the conceptual network. This process is especially efficient and the emerging concepts are particularly well-defined in places where the conceptual network is dense, well-determined and matched by a rich and structurally varied language. Language plays a very important role in the process of generating concepts, but it is rather a "channelling device", and therefore a passive factor. An active factor is still needed, that is a generator of concepts. Two such generators can be distinguished: primary and secondary.

The primary generator of concepts is the internal activity of the conceptual network, that is autonomous processes in the mind corresponding to broadly understood thinking. It can frequently work very efficiently, as long as it operates with a well-determined conceptual map and particular concepts, or, what is equivalent, a well-defined layer of language and linguistic names. Mathematics is the best example. In mathematics, there are highly refined concepts accompanied by language with a rich and ordered structure. This allows verification of statements, and therefore avoidance of arbitrariness, which greatly helps to specify newly formed concepts (by verification in this context we should understand a process which ensures that a given conceptual system is internally consistent "with a good approximation"). In other words, the conceptual network of mathe- 
matics is so well-specified locally, that the newly formed concepts within and on the basis of it are also well-specified. However, this verification is entirely internal in relation to the conceptual network. Also, new conceptual systems or theories in natural sciences, "humanities or philosophy emerge here by the operation of the "pure reason". This is the rationalistic way of the development of the conceptual network.

The external verification of concepts can be ensured only by the external world. This is the empirical way of the development of the conceptual network. The confrontation of concepts and conceptual maps with the world constitutes the secondary generator of concepts, and this is why we should assign such an important role to natural sciences in this process. Perhaps the discussed phenomenon is not "generation" in a strict sense, but at the level of the conceptual network such semantic distinctions are fading. Within the conceptual network of science various, frequently competing with each other, hypotheses and theories, equivalent to conceptual maps, are created. The emergence of new concepts can be also directly stimulated by observations and results of experiments (by conceptual maps underlying them). Subsequently, the conceptual sub-networks (maps) of theories and hypotheses are confronted with the external reality through new experiments and observations, based, however, on the hitherto existing and previously consolidated conceptual maps (theories). Validation, selection, elimination and/or modification of the conceptual maps of new theories and hypotheses takes place. Some of them are strengthened and temporarily verified, other - rejected instantly or with some finite delay. In this process, the verification of conceptual structures $=$ theories is much more severe and effective than only within the conceptual network itself. This is due to the lack of relativization by connotational relations. Contrary to the conceptual network, the external world of noumena can be regarded as "absolute" and "in-itself", although cognizable only to a limited extent. Even the exactness of mathematics and logic is not completely autonomous, as these disciplines use objects (such as a number, a point, a straight line, a set, implication) derived originally from the real world (or its projections on the everyday conceptual map) (Korzeniewski, 2013b, 2017). Thus, while the relation of strict denotation between the conceptual network and the real world is impossible, the facts of this world constitute points around which the conceptual network must tighten, if it is to keep contact with the world. And because of the requirement of the pragmatism of biological evolution, our conceptual network is somehow adapted to exploration of the external world. On the other hand, what was sufficient for our ancestors to hunt mammoths, now could fail in further exploration of the world, when natural selection has practically ceased to work, especially in highly-developed societies (apart from trivial cases of lethal mutations). In other words, the structure of the conceptual apparatus is definitely not pre-adapted by biological evolution to solve purely scientific or philosophical problems.

Moreover, our mind is directed not only by the cognitive drive, but also by other drives (e.g., self-preservation instinct, sexual drive or instinct of satisfying 
hunger, desire of richness and power, and generally - the striving to stimulate positively the reward system in the brain) pulling us away from the "objective truth" in the direction of mental comfort. However, the principal obstacle in reaching the "absolute" knowledge of the world is the connotative character of the conceptual network. On the other hand, the connotative mind is the only mind possible, and as such its functioning must involve contradictions and inconsistencies (Korzeniewski, 2010). Any denotational entity, e.g., a computer, based on stiff algorithms will remain in principle devoid of psyche and (self-)consciousness. We could, of course, model a connotational mind in a computer, but this procedure would have to consist in implementation into a computer program of the dynamic structure of functional connections between neurons within the neural network. However, such a mind would operate at decidedly higher level than the operating structure of the computer.

We can give yet another example of the necessity of internal contradictions in the mind created by biological evolution. Logical analysis will always show that "nothing has any meaning". In other words, it is impossible to justify rationally the meaning, value of anything, including one's life and cognitive activity. However, an individual deprived of any sense of meaning of life (motivation, intentionality) and value of various purposes in life is worthless from the perspective of biological evolution and will be eliminated by natural selection. A conscious entity, by the very fact of existing, falls into a logical contradiction. Therefore, it can emerge only in the process of evolution that has some autonomous "aims" (or rather mechanisms) and can possess only a connotative mind. However, the other (the first, actually) side of the coin is the fact that the human cognitive apparatus (and thus its conceptual network) is to some extent directed at examining the external world and processing the data coming from it. The good congruence of the conceptual network (at least in the pragmatic, instrumental sense) to the external world (especially to its aspects important from the point of view of natural selection) determined evolutionary success. This is why we regard natural sciences, which are sophisticated tools for examining this world equipped with appropriate, reliable methodology, as so important in the process of generating concepts.

Things are different in philosophy (and most humanities). If it is to create new concepts only on their own, then this process must be, in principle, not very efficient, and the created concepts are not much more meaningful than empty concepts. For, philosophy has at disposal neither a well-defined conceptual network (and thus well-defined language) and well-determined and separated concepts (and linguistic names), nor a direct contact with the world and ability to verify its conceptions. Thus, philosophy has neither the internal (rational), nor external (empirical) possibility to verify and adequately develop its concepts and conceptual network. It simply lacks the methodology of science, and in fact any reliable methodology. Therefore, contemporary philosophy is bound to assimilate concepts from natural and formal sciences, and this is practically the only opportunity for its real and fruitful development. In other words, science should be 
regarded as the main generator of (well determined, relatively sharp and well adhering to various aspects of reality) concepts (and paradigms) for philosophy. Instead of preceding science in the exploration of various aspects of reality, as philosophy was doing for millennia, it must now wait for science to form new concepts and new ways of grasping reality, which it could use. But the relationship between philosophy and science has not been entirely reversed. Philosophy, as a "semantic buffer" on the border of the semantic void, will always in some way precede science in the description of the world. Let us take, as an analogy, the example of biological succession. Just as on a bare rock first lichens appear and change the rock surface, making it available to other plants, which then gradually substitute them, so philosophy is preparing some semantic spaces (even if only by asking relevant questions), which then are taken up by science, clarifying and verifying concepts (and making more precise questions) provisionally formed by philosophy. Thus, we can speak about mutual stimulation. However, it is almost a truism to say that the centre of gravity has shifted decisively in the direction of science.

The confrontation of the conceptual network with the reality is a strong generator of new concepts. The incorporation of new concepts in relation to the existing ones, the "budding" of new concepts from the existing corpus of the conceptual network, determines the conceptual development of the conceptual network of science. As the conceptual network "gets denser" and begins to contain more better specified concepts and significative axes, new answers and new questions emerge, the existence of which cannot even be imagined on the basis of a simpler conceptual network. On the other hand, the concepts, which were previously well-established and intuitively perceived as clear and unambiguous, now become "fuzzy". Physics and neurophysiology provide good examples of the processes described above. In physics, the concept of matter, which was previously clear and obvious, now practically does not exist. It has been dismantled into the concepts of field intensity, equivalence with energy, particle-wave duality, and probability function (Korzeniewski, 2014). Einstein's general relativity is a great example of the phenomenon of relativism and relational nature of meanings in a conceptual system - the concepts of space, time, space-time curvature, matter, mass, gravity, speed, and energy are so formulated there that they explicitly have meanings only in relation to other concepts. If we removed one element, the whole structure would collapse. Thus, for example, the removal of matter (or energy) would change the structure of space-time dramatically. Similarly, in neurophysiology and neuropsychology, the concept of spirit (consciousness) is gaining attributes that have never been sensed before, and is losing its obviousness, unity and intuitive clarity. Physiological and molecular bases of neural signals transduction, perception, memory, and association processes, as well as the impact of external factors on the state of consciousness, consistently reduce the area reserved for the "spirit in its immanence", or "pure (self-)consciousness", and increasingly indicate the secondary character of consciousness in relation to matter (on the other hand, matter is received and perceived by us only through 
our consciousness, the conceptual network constituting it "substance") (Korzeniewski, 2014, 2015, 2017). Thus, the intuitively homogeneous picture of spirit as an integral and autonomous entity has been largely disassembled. And again, the original concept of consciousness is "budding" with derivative concepts, which "take with themselves" some of its content, part of its connections with other concepts. Of course, here science has not yet gone as far as in the case of the concept of matter (but also e.g., space and time) in physics. We still cannot break the very core of the mind - the sense of (self-)consciousness, or ego - into specific concepts. Will it ever be possible? I believe that it will happen to the extent that it did with matter: the very concept will slip through our fingers, and the name, not designating anymore anything absolute or indivisible, will become empty. One of the attempts to "break down" (self-)consciousness into simpler concepts was presented above and in previous publications (Korzeniewski, 2010, 2013a, 2015, 2017).

The differences between the primary generator and the secondary generator of concepts (and thus between the purely rationalistic internal activity of the conceptual network and its empirical confrontation with the external world) can be characterized in another way, with even bigger emphasis on the role of natural and formal sciences in the development of the conceptual network. Even in the best-specified conceptual maps, such as the conceptual map (underlying the language) of mathematics and logic, the meaning of concepts is defined only with some approximation, although the "semantic uncertainty" may be very small (Korzeniewski, 2014, 2015, 2017). Let us imagine that a given conceptual map is expanding and creating new concepts. On the basis of these new concepts, still other concepts develop. In this way, some "conceptual chains" emerge in the semantic space; these are strings of primary and derivative concepts in the conceptual network. Now, in such chains the indeterminacy of meaning of these concepts adds up as we move along a chain. Sufficiently long chains, even those composed of very well-defined concepts, will start to "bend", or "deviate" from some fixed line in the semantic space. Susceptibility to these "deviations" is a measure of "semantic uncertainty". Therefore, verification of a system's consistency in the primary generator of concepts is always only limited and local. For, if some conceptual chains develop independently of each other, without any external verification, from a conceptual nucleus (germ), for instance from the everyday conceptual map, then in sufficient distance from this nucleus they start to contradict one another (as an example, we can quote various religions and philosophies). Thus, in a global sense, the (purely internal) verification of the conceptual network structure is certainly insufficient in the case of the primary (purely rationalistic) concept generator.

It is different with the secondary (empirical) generator. In natural sciences, all newly emerging concepts or larger areas of the conceptual network (conceptual maps corresponding to scientific theories) are confronted with the external world immediately or with some finite delay. For instance, the Big Bang theory and the Steady State theory of the evolution of the universe coexisted for some 
time in the absence of evidence falsifying one of them. These competing theories only reflected philosophical inclinations of their advocates. However, the discovery of the microwave background radiation (and many other phenomena) settled the issue in favor of the Big Bang theory. Thus, the external objective world in a sense determines the lines in the semantic space, which the conceptual chains must "conform" to. Of course, the mutual congruence of these lines and chains is never perfect. However, semantic indeterminacy does not add up as the chain develops, but remains more or less constant. Thus, assuming that the external world is non-contradictory, it imposes this non-contradictoriness (of course, only imperfectly and approximately) on the structure of the conceptual network. First of all, this applies directly to the conceptual map of natural sciences (the everyday conceptual map has long been, to some limited extent, agreed with the external world). Indirectly, however, philosophy and everyday spheres are also under this influence. Thus, the entire conceptual network not only could not have emerged without the external world, which is a rather trivial statement, but this world is still necessary for its rational development as a whole. And because currently only natural sciences have adequate methodology (experiments, observations, mathematical models, validation) allowing the estimation of the relative degree of correspondence between the structure of the real world and the structure of the conceptual network, they offer the main opportunity for the development of philosophy.

It is a different matter with broadly understood culture and humanities (and philosophical systems drawing from them). First of all, they lack the external discussed above-verification of their claims, and their internal verification is very weak. In other words, they do not possess any reliable methodology that would allow not only to collect individual facts, but also to integrate them into a reliable, internally consistent and fruitful theory with the ability of prediction of new facts. The evolution of natural sciences created in different cultures must converge - this convergence is a derivative of the congruence of the structures of these sciences to the structures of the "univocal" world. Therefore, this trait of the evolution of the structure of the conceptual network of science escapes to a large extent the Kuhn's relativism (Kuhn, 1962). The methodology of natural sciences is able to eliminate (sooner or later) any significant deviations of the structure of science (currently accepted scientific theories) from the structure of the external reality. Thus, it operates on the principle of the negative feedback.

In contrast, the development of most of culture (including humanities and philosophies based on them) consists in reinforcing some accidentally chosen direction and further going along this path. Culture determines (to some extent) the course of its further evolution (fixes the boundaries of further development) through the already possessed structure. Thus, it operates on the principle of the positive feedback. The further a given culture has gone along a given route of development, the more decidedly it follows this route. As the direction of development is "chosen" in a given culture by chance, evolution of different cultures is divergent. The humanities, studying culture, create it at the same time. For 
natural sciences, the object of their studies exists independently of them. Culture and the humanities (and many philosophies) frequently create by themselves the object of their studies. Science can be compared to a convolvulus that winds around a wooden pole (=reality). On the other hand, a complex of various cultures (including philosophies) resembles a bush bifurcating vigorously in all directions. When the disciplines of humanities study culture, they simultaneously create it. In natural sciences, a subject of their research exists independently of them. Culture and humanities create the subject of their research themselves. They start from the biological constitution of human beings (and from the physical nature of the external world), but this is not their object of interest they are interested in what is built on it. The learning of new structures is here essentially indistinguishable from creating them. This creation has no limits, in the sense that the created structures do not have to conform to anything except themselves. There is complete freedom and arbitrariness. Culture needs only to have "some consideration" for the biological constitution of human beings and physical realities of the world. For instance, it is limited by the law of gravity or by human mortality (although it is not difficult to find within it systems that even circumvent these basic facts). However, apart from this necessary minimum, it does not have any points of contact with the objective world.

Two independently developing physics (e.g., in two different cultures or on two different planets) might have different starting points (within culture and language) and might use different concepts. However, their structure would have to conform-better or worse-to the structure of the objective world. And as this world, however it is understood, should be regarded as unambiguous, they would also have to conform to each other on the basis of transitivity: if $\mathrm{A}$ is congruent to $\mathrm{C}$, and $\mathrm{B}$ is congruent to $\mathrm{C}$, then $\mathrm{A}$ is congruent to $\mathrm{B}$. Thus, the answer to the question of whether it is possible to have two different physics with two different underlying mathematical structures, is: yes and no. Yes, because they can start from different concepts taken from everyday language and experience, use different starting assumptions, and be (to some extent) differently directed by stochastic processes. No, because the structures built within them must conform to the structure of the objective world, and therefore to each other. This protects natural sciences against arbitrariness. Humanities tacitly accept the fact of existence of the objective world, but they focus their attention on human culture that is developing to a large extent in an accidental way. This property, combined with the lack of verifiability and any reliable methodology, makes humanities and culture a very uncertain base for philosophy.

During the evolution of the conceptual network of culture (Korzeniewski, 2015 , 2017) there can be seen a clear tendency to absolutize linguistic names and their underlying meanings (concepts), and to attribute real existence to their designates (Korzeniewski, 2013b). The real world, continuous in its essence, is, when seen through the prism of language, "sliced" into sharply separated pieces, organized in a discrete way, pushed in into the corset of absolute categories (Korzeniewski, 2013b). Linguistic names arbitrary demarcate discrete objects, 
events and sets within the reality, which are "naturally" absent there. Inevitably, the names and sentences of language correspond only roughly and approximately to some aspects of the world, and attribute to the world features that are only and exclusively features of language. The segregation of the phenomena perceived by our senses and processed by integrative structures in the sensory cortex (that already carry out a preliminary categorization and "discretization") into different discrete semantic compartments facilitates excellently our manipulation with the accessible information, and therefore the functioning and development of the conceptual network. However, this process results at the same time in a significant distortion, deformation of the representation (picture) of the world formed in within the conceptual network (Korzeniewski, 2013b).

Culture as a whole and most of philosophy has much in common with magic and religion (Korzeniewski, 2013b). All these disciplines are characterized by far-going autonomization, absolutization and "realization" of words of language. For instance, magic not only establishes a kind of necessity of the relation between names and their designates, completely ignoring its accidental character, but also endows words a performative power. The esoteric and necessary character of names also manifests itself in religion. For instance, Jehovah's Witnesses or Muslims take pride in their knowledge of the true name of God. On the other hand, philosophy has a strong tendency to absolutize such names (and concepts) as spirit, matter, monads, four elements, but also, for instance, concepts within the philosophy of science, such as scientific paradigms (Kuhn, 1962) or research programmes (Lakatos, 1970), and so forth. However, the world is not discrete like language, but continuous like the conceptual network. Therefore, recognition of the "fact-creating" role of language has a fundamental significance for epistemology (Korzeniewski, 2013b), but also for the philosophy of nature.

Of course, also here science plays a crucial role. As it was mentioned above, physics has decomposed the concept of matter into field equations, wave-functions, equivalence with energy, electro-magnetic, gravitational and nuclear forces as well as other concepts, being as much real objects as products of our mind, so that matter has become in fact only an empty name (Korzeniewski, 2014). The process of de-composition of the concept of (self-)consciousness (spirit) into other concepts performed by neurophysiology is also significant, although it has not advanced that far. Nevertheless, the apparently sharp opposition between the mind and matter seems to vanish gradually, being "dissolved" in the sea of the surrounding concepts. For instance, in theoretical physics we cannot univocally decide, what is the "objective" aspect or property of the external world, that is broadly understood matter, and what is the subjective product of our mind, our conceptual network! Matter seems to be to an increasing extent (at least within theoretical physics) a product of the human mind, while all known evidences indicate that the mind emerges from the functioning of (sufficiently and appropriately complex) material systems (human brains). As the development of science proceeds, the mind-matter opposition is still less and less sharp and well-defined 
(Korzeniewski, 2015, 2017). The same applies to many other concepts and problems, with which philosophy struggled for centuries, and which were shown by science as empty or apparent. Therefore science, by escaping from the conventionality of culture, enables us to de-mythologize many aspects of our culture, especially philosophy. At the same time, science (especially neurophysiology and psychology) suggests that the conceptual network is a more adequate tool for formation of a relatively faithful representation of the world than language. Language, being anyway a part of the conceptual network, is a very efficient tool that allows an easy, but far from perfect, operation and manipulation with concepts within the entire network. However, this does not mean that human (including scientific) thinking has a linguistic nature and occurs primarily on the linguistic level (Korzeniewski, 2013b, 2015, 2017).

\section{Summary}

According to the Conceptual-Network-Based Philosophy of Science (CNBPS), the conceptual network of science, underlaid by an appropriate neural sub-network in the human brain, is composed of conceptual maps, sub-maps and (complexes of) concepts corresponding to scientific disciplines, theories, hypotheses and "objects". Particular concepts within the conceptional network mean by connotation, relation to each other. Novel scientific theories (their conceptual maps) are created (and preliminarily evaluated) within individual networks of particular scientists and afterwards possibly accepted, consolidated an inbuilt into the collective conceptual network of science or rejected by the scientific society constituting the "carrier" of this collective conceptual network.

The appearance of new theories consists in creation of new, differently structured fragments (conceptual maps) of the conceptual network of science on the basis of the already existing conceptual network as well as of sensations and concept complexes related to new observations and experiments. New conceptual maps (scientific theories) originate from "conceptual germs" that are partly independent on (lie outside of) the conceptual maps of old theories. The conceptual network of science develops by splitting of old concepts into new concepts, adding new concepts on the basis of the existing network, further determination of the already existing concepts and elimination of the concepts that do not fit to the evolving structure of the network. New conceptual maps and sub-maps of scientific theories and hypotheses can be created on the basis of the existing conceptual network of a given scientific discipline, "bud" from it. The primary (empirical) source of new concepts is incoming sensations. These sensations can constitute elements of observations or experiments. However, their interpretation and understanding are conditioned by the already existing structure of the conceptual network of science. New sensations can be in turn inbuilt into the existing conceptual maps of scientific theories as new concepts or their complexes. Sensations can be further associated into primary and secondary concepts within the conceptual network of science. Primary concepts represent particular objects, events and processes in the (external or internal), while sec- 
ondary concepts represent general laws, categories, regularities and universal beings. The secondary (rationalistic) source of new concepts and mechanism of the development of the conceptual network of science is the autonomous activity of the individual conceptual. These are broadly understood thinking processes consisting in the functioning and modification of existing concepts as well as formation (or elimination) of new concepts within the conceptual network on the basis of the existing ones.

The collective, inter-subjective conceptual network of science as a social phenomenon can be regarded as a part (sub-network) of the conceptual network of broadly-understood culture. It constitutes a derivative of individual conceptual networks, vaguer and less specified than them, corresponding to the Popper's third world. Creation of scientific theories occurs initially within the individual conceptual networks of particular scientists, while their common acceptance or rejection-in the collective conceptual network of science. It constitutes a sum, average or "resultant" of the individual networks of particular researches, but at the same time it co-shapes reciprocally these networks. Therefore, the bi-directional interactions between the individual conceptual networks of scientists and the collective conceptual network of science are crucial for the development of science. Invention of novel theories (conceptual maps of science) constitutes a result of the autonomous activity (equivalent to thinking processes) of the individual conceptual network, and the neural network underlying it. The confrontation of conceptual maps of new scientific theories and hypotheses with the existing conceptual maps of theories, observations and experiments is carried out by individual scientists and teams of scientists, and its results belong to the collective conceptual network of science. Similarly, the conceptual maps of new theories are being referred to sensations and concepts related to new observations and experiments, but also to various existing, well established theories, and the whole scientific society decides whether the former fits satisfactorily to the latter or not. The conceptual maps of new consolidated theories undergo incorporation into the collective conceptual network of science. The development of science in both networks consists in the analysis and comparison of various conceptual maps, extraction of repetitive patterns in different complexes of secondary concepts, formation of new, differently-structured conceptual maps (theories), relating them to the already existing conceptual maps as well as known and currently gathered facts (observations and experiments with sensations and concept complexes underlying them). Such differently-structured conceptual maps of new theories are able to infer the conceptual and "real" structures of new, still undiscovered aspects, objects and processes of the physical world and thus predict their existence.

Scientific theories cannot be either entirely true or false. The structures of conceptual maps of scientific theories only can adhere better or worse to the structure of certain aspects of the reality. Their logical truth value is never exactly 0 or 1, but is situated somewhere between 0 and 1. A new, better, more progressive theory that replaces an old, less advanced theory is simply underlaid by 
a conceptual map with a structure that adheres better to the structure of a greater number of various aspects of the (external and internal) world, than the structure of the conceptual map of the old theory. The truth value of the new theory is relatively closer to 1 , than the truth value of the old theory. However, it is not possible to estimate the distance of the truth value of a given theory from 1 in absolute terms. The old theory replaced by a new theory is by no means falsified. The structure of its conceptual map simply adheres worse to less numerous aspects of the reality. Nevertheless, this adherence is still relatively good, as the old theory worked pretty well for quite a long time. The conceptual structure of the new theory, or at least some of its fragments, must be to some degree congruent to, isomorphic with the conceptual structure of the old theory, as both adhere better or worse to the real structure of the same "objective" world.

The conceptual network of science in general and the conceptual maps of its theories, in particular, by no means aim to reach any ultimate and absolute truth. The conceptual structure of scientific theories can only adhere, in an on principle imperfect and approximate way, to the structure of (various aspects of) the real world. The conceptual network entwines the reality as the spider web entwines a stony sculpture. The structure of the web conforms to some extend to the structure of the sculpture surface, but their congruence is never perfect, and the "substance" of the spider web is of completely different nature, than the "substance" of the stony sculpture. The system of meanings in the conceptual network of science is relational and connotative, and particular (complexes of) concepts do not designate directly any noumena of the external world. There takes place a real, although imperfect, progress in science towards the truth, but it is not possible to reach the absolute truth, the very immanent essence of the world. Therefore, this cognitive position of the Conceptual-Network-Based Philosophy of Science (CNBPS) proposed in this work can be called the semi-representative realism.

\section{Conflicts of Interest}

The author declares no conflicts of interest regarding the publication of this paper.

\section{References}

Ayer, A. J. (1959). Logical Positivism. Glencoe: Free Press.

Chalmers, A. F. (1999). What Is This Thing Called Science? St. Lucia: Queensland University Press.

Chalmers, D. J. (1997). The Conscious Mind: In Search of a Fundamental Theory (Philosophy of Mind). Oxford: Oxford University Press.

Greene, B. (2005). The Fabric of the Cosmos, Space, Time and the Texture of Reality. New York: Vintage.

Hume, D. (1975). A Treatise of Human Nature. Oxford: Clarendon Press.

Kant, I. (1999). Critique of Pure Reason. Cambridge: Cambridge University Press.

Korzeniewski, B. (2010). From Neurons to Self-Consciousness. How the Brain Generates 
the Mind. Amherst, MA: Prometheus Books.

Korzeniewski, B. (2013a). Formal Similarities between Cybernetic Definition of Life and Cybernetic Model of Self-Consciousness: Universal Definition/Model of Individual. Open Journal of Philosophy, 3, 314-328. https://doi.org/10.4236/ojpp.2013.32049

Korzeniewski, B. (2013b). Magic of Language. Open Journal of Philosophy, 3, 455-465. https://doi.org/10.4236/ojpp.2013.34067

Korzeniewski, B. (2014). Philosophy of Conceptual Network. Open Journal of Philosophy, 4, 451-491. https://doi.org/10.4236/ojpp.2014.44050

Korzeniewski, B. (2015). Mind-Body Problem: Does Complexity Exist Objectively? Open Journal of Philosophy, 5, 351-364. https://doi.org/10.4236/ojpp.2015.56043

Korzeniewski, B. (2016). Freezing of Reality: Is Flow of Time Real? Open Journal of Philosophy, 6, 256-264. https://doi.org/10.4236/ojpp.2016.63025

Korzeniewski, B. (2017). Does Matter Matter? Should We Mind the Mind? Can Philosophy Be Reduced to Neurophysiology? Open Journal of Philosophy, 7, 265-328. https://doi.org/10.4236/ojpp.2017.73017

Kuhn, T. S. (1962). The Structure of Scientific Revolutions. Chicago, IL: University of Chicago Press.

Lakatos, I. (1970). Falsification and the Methodology of Scientific Research Programmes. In I. Lakatos, \& A. Musgrave (Eds.), Criticism and the Growth of Knowledge (pp. 91-195). Cambridge: Cambridge University Press. https://doi.org/10.1017/CBO9781139171434.009

Penrose, R. (2002). The Emperor's New Mind: Concerning Computers, Minds, and the Laws of Physics. Oxford: Oxford University Press.

Popper, K. (1968). Logic of Scientific Discovery. London: Hutchinson.

Popper, K. (1978). Three Worlds. The Tanner Lecture on Human Values. Ann Arbor, MI: The University of Michigan.

Preston, A. Analytic Philosophy. Internet Encyclopedia of Philosophy. https://www.iep.utm.edu/analytic

Quine, W. V. O. (1960). Word and Object. Cambridge, MA: MIT Press.

Russell, B. (1912). Problems of Philosophy. Oxford: Oxford University Press.

Salmon, W. C. (1975). The Foundations of Scientific Inference. Pittsburgh, PA: Pittsburgh University Press.

Sapir, E. (1921). Language: An Introduction to the Study of Speech. New York: Harcourt, Brace \& Co.

Tarski, A. (1935). The Concept of Truth in Formalized Languages. In A. Tarski (Ed.), Logic, Semantics, Metamathematics (pp. 152-278). Indianapolis, IN: Hackett.

Weber, M. (2001). The Protestant Ethic and the Spirit of Capitalism. London and New York: Routlege.

Whorf, B. L. (1940). Science and Linguistics. Technology Review, 42, 229-231.

Wittgenstein, L. (1922). Tractatus Logico-Philosophicus. London: Kegan Paul. 